\title{
ANEMIA COMO FACTOR PRONÓSTICO EN PACIENTES CON CÁNCER
}

\author{
Victor Paitan 1,a, Cindy Alcarraz 1,a , Angela Leonardo ${ }^{1, b}$, Guillermo Valencia ${ }^{1, b}$, Raúl Mantilla ${ }^{1, c}$, Zaida Morante $^{1, a}$, \\ Teodoro J. Oscanoa ${ }^{2,3, d}$, Luis Mas ${ }^{1, a}$
}

\begin{abstract}
RESUMEN
Objetivos. Determinar la frecuencia y el valor pronóstico de la anemia en pacientes con cáncer atendidos en el Instituto Nacional de Enfermedades Neoplásicas (INEN) entre enero y abril del 2010. Materiales y métodos. Se consideró anemia en varones cuando la hemoglobina fue $<13 \mathrm{~g} / \mathrm{dL}$, y en mujeres cuando fue $<12 \mathrm{~g} / \mathrm{dL}$. Para determinar asociaciones se usó la prueba Chi-cuadrado. Para el análisis de las curvas de sobrevida se usó el estimador de Kaplan-Meier y log rank test. Resultados. 772 pacientes fueron incluidos; 584 (75,7\%) tuvieron tumores sólidos y $188(24,3 \%)$ neoplasias hematológicas. Se diagnóstico anemia en 359 (46,5\%) pacientes, en $124(66,0 \%)$ neoplasias hematológicas, y en 235 $(40,2 \%)$ neoplasias sólidas. Las neoplasias hematológicas con mayor frecuencia de anemia fueron la leucemia mieloide crónica, las leucemias agudas, y el mieloma múltiple (100\%, 92,5\% y 60\%; respectivamente) y en el grupo de neoplasias sólidas fueron los cánceres de origen: gastrointestinal, ginecológico, y urológico (62\%, 52,1\% y 45\%; respectivamente). Recibieron transfusiones 204 pacientes $(26,4 \%)$. En 762 pacientes se encontró una diferencia en la sobrevida global entre los grupos sin y con presencia de anemia, estimándose a los cinco años en $62 \%$ y $47 \%$ respectivamente $(p<0,001)$, además se encontraron diferencias en la sobrevida global para el subgrupo de tumores sólidos $(p=0,002)$ y neoplasias hematológicas $(p=0,007)$. Conclusiones. La anemia es frecuente en pacientes con cáncer y su presencia determina un factor pronóstico independiente en la sobrevida global.
\end{abstract}

Palabras clave: Anemia; Neoplasias; Supervivencia; Perú; Pronóstico (fuente: DeCS BIREME).

\section{ANEMIA AS A PROGNOSTIC FACTOR IN CANCER PATIENTS}

\begin{abstract}
Objective. To determine the frequency and prognostic value of anemia in cancer patients receiving care at the National Institute of Neoplastic Diseases (Instituto Nacional de Enfermedades Neoplásicas - INEN) between January and April of 2010. Materials and Methods. Anemia was considered for men with hemoglobin levels at $<13 \mathrm{~g} / \mathrm{dL}$; and for women, at $<12 \mathrm{~g} / \mathrm{dL}$. Associations between qualitative features were assessed with a Chi-square test. Kaplan-Meier estimator was used for the analysis of the survival curves, and differences between the curves were performed with the log-rank test. Results. 772 patients were included; $584(75.7 \%)$ had solid tumors and 188 (24.3\%) had hematologic malignancies. Anemia was diagnosed in 359 patients (46.5\%); hematologic malignancies in 127 patients (67.6\%); and solid neoplasms in $235(40.2 \%)$. Hematologic malignancies with the highest frequency of anemia were chronic myeloid leukemia, acute leukemias, and multiple myeloma (100\%, $92.5 \%$ and $60 \%$, respectively); and were cancer of gastrointestinal, gynecological, and urological origin were in the group of solid neoplasms $(62 \%, 52.1 \%$ and $45 \%$, respectively). Two hundred and four (204) patients (26.4\%) were transfused. In 762 patients, a significant difference in overall survival was found between groups with and without anemia, estimated at 5 years in $62 \%$ and $47 \%$ respectively $(p<0.001)$. In the solid tumor subgroup $(p=0.002)$, and the hematological malignancies subgroup $(p=0.007)$, such association was also found. Conclusions. Anemia is common in cancer patients, and its presence determines an independent prognostic factor in overall survival.
\end{abstract}

Keywords: Anemia; Neoplasms; Survival; Peru; Prognostic. (source: MeSH NLM).

\footnotetext{
1 Departamento de Medicina Oncológica, Instituto Nacional de Enfermedades Neoplásicas. Lima, Perú.

Departamento de Medicina Interna, Hospital Nacional Guillermo Almenara Irigoyen. Lima, Perú.

3 Instituto de Investigación de la Facultad de Medicina, Universidad de San Martin de Porres. Lima, Perú.

a Médico Oncólogo Clínico; ${ }^{\mathrm{b}}$ Médico Residente; ${ }^{\mathrm{c}}$ Bachiller en Bioestadística; ${ }^{\mathrm{d}}$ Médico internista

Recibido: 21/10/2017 Aprobado: 18/04/2018 En línea: 28/06/2018
}

Citar como: Paitan V, Alcarraz C, Leonardo A, Valencia G, Mantilla R, Morante Z, et al. Anemia como factor pronóstico en pacientes con cáncer. Rev Peru Med Exp Salud Publica. 2018;35(2): 250-8. doi: 10.17843/rpmesp.2018.352.3171. 


\section{INTRODUCCIÓN}

La anemia es un indicador de pobre salud y nutrición a nivel global. La causa de anemia es multifactorial, siendo más frecuente por carencias nutricionales además de estados de inflamación aguda y crónica, las parasitosis y enfermedades hereditarias o adquiridas también afectan la síntesis de hemoglobina y la producción de los eritrocitos ${ }^{(1)}$. La prevalencia de anemia a nivel global está cerca de $24,8 \%$, llegando a afectar a 1620 millones de personas. A nivel de Latinoamérica la prevalencia en mujeres en edad fértil no embarazadas es $23,5 \%$, y a nivel de Perú está en $40,4 \%$, por lo cual, es considerado un grave problema de salud pública. ${ }^{(2)}$

La frecuencia de anemia en pacientes con cáncer varía considerablemente, probablemente debido a diferencias entre grupos poblacionales y métodos de diagnóstico. Se ha descrito a nivel mundial la prevalencia de anemia entre el 30 al $90 \%$, dicha variabilidad se explica por las diferentes definiciones de anemia, tipos de cáncer y estadios. Además, su presencia está relacionada a una menor sobrevida, menor control local de la enfermedad y peor calidad de vida. En Europa también se ha encontrado la presencia de anemia al diagnóstico en $39 \%$ para todos los tipos de cáncer (3). En Perú se encontró una frecuencia de anemia de 55,7\% en mujeres menores de 35 años con cáncer cervical ${ }^{(4)}$.

La anemia puede ocurrir como un efecto directo del cáncer (sangrado asociado al tumor, hemólisis, hiperesplenismo con hemofagocitosis, infiltración de la médula ósea), como resultado del tratamiento (quimioterapia, radioterapia, inhibidos de tirosinkinasa, anticuerpos monoclonales) o a través de factores químicos producidos por el cáncer (citoquinas inflamatorias como interferón gamma e interleuquina y factor de necrosis tumoral alfa pueden influir en la producción de eritropoyetina, e impedir el metabolismo del hierro) ${ }^{(2,3,5,6)}$. Tan importante como su diagnóstico, han sido las intervenciones para reducir los niveles de anemia, por ejemplo, los agentes estimulantes de eritropoyetina cuyo uso es controversial por los riesgos de morbimortalidad asociados. El dosaje de hierro, así como su uso en terapia endovenosa para reducir los porcentajes de transfusión han sido también estudiados ${ }^{(7,8)}$.

La presencia de anemia al inicio del tratamiento ha sido ampliamente estudiada, tanto en la respuesta al tratamiento, en la progresión de enfermedad, así como una menor sobrevida, mayor mortalidad, en la efectividad al tratamiento ya sea con radioterapia o quimioterapia, y en puntuaciones de calidad de vida, siendo considerado un factor pronóstico independiente de sobrevida en pacientes oncológicos. El incremento del riesgo de muerte de pacientes oncológicos con anemia se ha calculado en un $65 \%{ }^{(2,9-11)}$; además, la hipoxia del tumor por anemia conlleva a angiogénesis; por tanto, su corrección tiene una mejor sobrevida y respuesta ${ }^{(12)}$.

\section{MENSAJES CLAVE}

Motivación para realizar el estudio. La anemia relacionada al cáncer es un fenómeno bastante frecuente y generalmente infravalorado al momento de su diagnóstico en pacientes diagnosticados de cáncer. Su frecuencia y su relación con la sobrevida global como factor pronóstico no ha sido descrito en el Perú.

Principales hallazgos. La frecuencia de anemia fue de $46,5 \%$. La sobrevida global fue mejor en aquellos pacientes que no tenían anemia al momento del diagnóstico.

Implicancias. La anemia en pacientes con cáncer necesita de medidas de intervención. Se hacen necesarios estudios prospectivos en nuestra población.

La calidad de vida está relacionada positivamente con los niveles de hemoglobina ${ }^{(13-16)}$. En ancianos la anemia está asociada a una menor tasa de sobrevida, es considerada un factor de riesgo independiente para muerte, mayor deterioro del estado funcional, complicaciones cardiovasculares y del sistema nervioso central, así como falta de respuesta al tratamiento ${ }^{(17)}$. Asimismo, la anemia fue asociada a una peor respuesta patológica a la quimioterapia neoadyuvante en pacientes con cáncer de mama, así como incremento de la tasa de recurrencia y mortalidad, y en linfomas de Hodgkin como parte de índices de puntuación pronósticos para determinar tasas de progresión de enfermedad y sobrevida global ${ }^{(18,19)}$.

La presencia de anemia en pacientes con cáncer supone una asociación importante que ha sido demostrado en múltiples estudios; sin embargo, no existen estudios a nivel local o regional que puedan validar esta información. El presente estudio busca determinar la frecuencia de anemia y su valor como factor pronóstico en pacientes con cáncer que ingresaron al Departamento de Oncología Médica del Instituto Nacional de Enfermedades Neoplásicas.

\section{MATERIALES Y MÉTODOS}

\section{DISEÑO Y POBLACIÓN DE ESTUDIO}

Es un estudio longitudinal retrospectivo. Casos nuevos de cáncer registrados en el Instituto Nacional de Enfermedades Neoplásicas durante enero y abril del 2010, con un seguimiento de 5 años.

\section{CRITERIOS DE SELECCIÓN}

Se incluyeron pacientes mayores de 14 años con diagnóstico de cáncer confirmado histopatológicamente y que tuvieron hemograma basal. Se excluyeron pacientes con síndromes mieoloproliferativos/mielodisplásicos, neoplasias benignas, en gestación o que fueron tratados previamente en otra institución.

\section{VARIABLES}

La variable edad se midió en años desde la fecha de nacimiento hasta la fecha de ingreso a la institución. La 
variable sexo fue sacada de la hoja de ingreso de acuerdo a la información del documento nacional de identidad (DNI). Las comorbilidades se definieron como toda aquella patología no oncológica obtenida de la historia clínica del paciente, que haya sido diagnosticado previamente en otra o en la misma institución, que esté recibiendo un tratamiento regular, llámese hipertensión arterial, diabetes mellitus, hipotiroidismo, etc. La variable tipo de tumor, se define de acuerdo a la histología de cada cáncer siendo de tipo hematológico: leucemias, linfomas, y mielomas múltiples; las neoplasias sólidas son todos los demás tipos de tumores.

La variable anemia se definió como valores de hemoglobina debajo del normal establecido de acuerdo a la escala de la Organización Mundial de la Salud (OMS), en mujeres $\geq 12$ $\mathrm{g} / \mathrm{dL}$ y en varones $\geq 13 \mathrm{~g} / \mathrm{dL}$ fueron considerados normales, asimismo la graduación de leve fue entre el limite normal inferior y $11 \mathrm{~g} / \mathrm{dL}$, para moderada entre 10,9 y $8 \mathrm{~g} / \mathrm{dL}$ y para severa $<8 \mathrm{~g} / \mathrm{dL}{ }^{(1)}$. Los valores normales de acuerdo al volumen corpuscular medio fueron de $83-96 \mathrm{fL}^{(20)}$. Los valores de hemoglobina y demás constantes se solicitaron al momento de la admisión del paciente al hospital como parte del plan de trabajo, cuyos resultados son de acceso directo con el uso del sistema operativo interno del hospital (SISINEN). La medición estuvo a cargo del departamento de Laboratorio y Hematología Clínica del INEN mediante XE-2100 ${ }^{\mathrm{TM}}$ Automated Hematology System - Sysmex, durante el periodo de evaluación no hubo variaciones en el equipo utilizado, el mismo que fue sometido a control de calidad y calibración diaria antes del procesamiento de las pruebas y a mantenimiento anual. El tiempo de diagnóstico de anemia fue calculado entre la diferencia de fecha de primer hemograma completo realizado en laboratorio y la fecha de ingreso a la institución (promedio dos días).

La variable estadio clínico para tumores sólidos se determinó de acuerdo al American Joint Commitee on Cancer 7th edition (AJCC) ${ }^{(21)}$

La variable transfusiones se refiere a la transfusión de un mínimo de un paquete globular realizada durante el periodo de ingreso de los pacientes (enero - abril 2010), esta información fue recabada del SISINEN.

La sobreviva global fue medida en tiempo desde la fecha de ingreso hasta su deceso sea o no en la institución, confirmada a través de la RENIEC en coordinación con el Departamento de Estadística del INEN.

\section{ANÁLISIS ESTADÍSTICO}

Se realizó un análisis descriptivo de la información a través de frecuencias, porcentajes y medidas resumen (promedio, mediana, rango). Asociaciones entre características cualitativas con la anemia se evaluaron con la prueba Chicuadrado, aplicándose la corrección de Yates, según correspondía. Diferencias entre los grupos de pacientes con y sin anemia respecto a variables cuantitativas se evaluaron con la prueba t para muestras independientes o su correspondiente prueba no paramétrica (luego de una evaluación de normalidad de la variable cuantitativa). Para la estimación de la sobrevida global, el tiempo de seguimiento comprendió desde la fecha de ingreso hasta la fecha de muerte (evento de interés) o la fecha de último control. En la estimación de las curvas de sobrevida se usó el método de Kaplan-Meier y diferencias entre las curvas se probó con el log-rank test. Un valor $p<0,05$ se consideró para una diferencia significativa. El valor pronóstico de la anemia, ajustado por otras características, se evaluó a través de un modelo de regresión de Cox multivariado, expresándose la medida de la fuerza de asociación a través de hazard ratio (HR) con su respectivo intervalo de confianza al 95\%. Se usó el programa estadístico $R$ (R Core Team 2017). R: A language and environment for statistical computing. $R$ Foundation for Statistical Computing, Vienna, Austria. URL https://www.R-project.org/).

\section{ASPECTOS ÉTICOS}

El estudio fue aprobado por el Comité Institucional de Ética en Investigación del Instituto Nacional de Enfermedades Neoplásicas (INEN) del Perú. Se mantuvo confidencialidad de los datos obtenidos de las historias clínicas.

\section{RESULTADOS}

Entre enero a abril del 2010, ingresaron un total de 928 pacientes al departamento de Medicina del INEN, cumplieron los criterios de selección, un total de 772 pacientes (Figura 1). Del total, 359 (46,5\%) presentaban anemia al ingreso a la institución. La mediana de edad fue de 54 años (14 a 93). La mediana del valor de hemoglobina fue de $12,5 \mathrm{~g} /$ $\mathrm{dL}(3,4$ a 18,7$)$. El valor promedio del hematocrito fue $37,8 \%$ $(9,6-57,2)$ y de las constantes corpusculares: VCM, HCM, y CHCM fue de 87,7 fL (40-116), 29 pG (15-39,6), y 328 g/dL (31,4-362). 510 mujeres fueron evaluadas $(66,1 \%)$, y 262 varones (33,9\%). Del total de mujeres, 42,2\% $(n=215)$ presentó anemia, siendo leve en 40,4\% ( $n=87)$, moderada en $44,7 \%(n=96)$ y severa en $14,9 \%(n=32)$. Del total de varones, $55,0 \%$ presentó anemia $(n=144)$, siendo leve en $52,1 \%(n=75)$, moderada en $34,0 \%(n=49)$ y severa en $13,9 \%(n=20)$.

La razón hombre/mujer fue de 1 a 1,9. 659 pacientes $(85,4 \%)$ no tenían antecedente de enfermedades crónicas, y $113(14,6 \%)$ pacientes presentan alguna comorbilidad, siendo anemia el 48,7\% ( $n=55)$. Aquellos menores de 60 años sumaron 491 pacientes $(63,6 \%)$ e igual o mayores a 60 fueron 281 (36,4\%), del primer grupo presentaron anemia 41,3\% ( $n=203)$, y del segundo grupo 39,9\% $(n=112)$. Del grupo de tumores sólidos $(n=584)$, se obtuvo en el estadio I 42 pacientes, estadio II 107 pacientes, estadio III 230 pacientes, y estadio IV 205 pacientes, la frecuencia de 


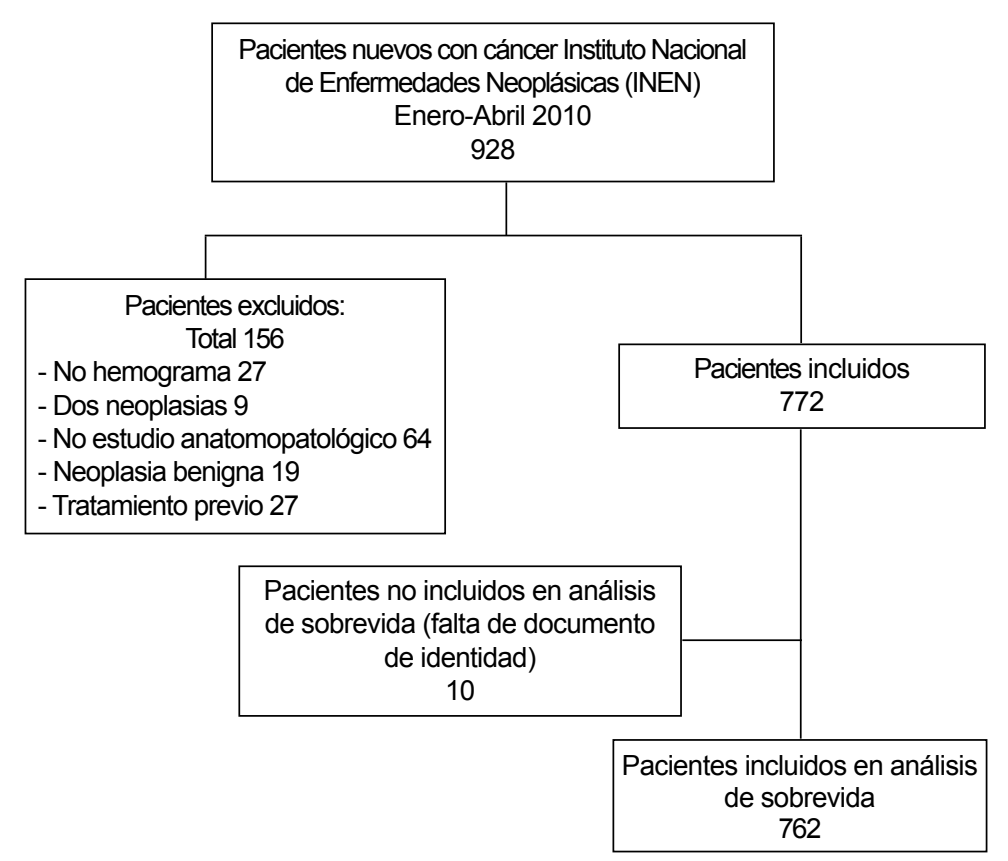

Figura 1. Flujograma de selección de pacientes

anemia en cada uno de los grupos fue: $26,2 \%(n=11), 37,3 \%$ $(n=40), 39,6 \%(n=91)$, y $45,4 \%(n=93)$. De acuerdo al valor de constantes corpusculares, la anemia microcítica fue de $34,0 \%(n=122)$, normocítica de $57,4 \%(n=206)$, macrocítica de $7,5 \%(n=27)$, y sin información en $1,11 \%(n=4)$. Acorde a la severidad de la anemia, fue más frecuente la anemia leve en $45,1 \%$ ( $n=162$ ), seguida de anemia moderada $40,4 \%$ $(n=145)$ y anemia severa en $14,5 \%(n=52)$ (Tabla 1$)$.

El grupo de neoplasias hematológicas estuvo conformado por 188 pacientes $(24,3 \%$ ) y el grupo de tumores sólidos por 584 pacientes $(75,7 \%)$, la presencia de anemia fue $66,0 \%$ $(n=124)$ y $40,2 \%(n=235)$ para cada grupo. Los seis tipos de cáncer más frecuentes fueron: mama, linfoma no Hodgkin, pulmón, cérvix, estómago y colón, siendo los porcentajes de anemia respectivamente: $26,3 \%, 54,9 \%, 25,5 \%, 57,5 \%$, $63,2 \%$ y $57,9 \%$. Las neoplasias más anemizantes fueron la leucemia mieloide crónica en $100 \%(n=11)$, leucemias agudas en $92,5 \%$ ( $n=37$ ), y el mieloma múltiple en $60 \%$ $(n=10)$. En el grupo de neoplasias hematológicas, las más anemizantes fueron la leucemia; así mismo, en el grupo de neoplasias sólidas fueron: neoplasias gastrointestinales en $62 \%(n=85)$, ginecológicas en $52,1 \%(n=37)$, y urológicas en $45 \%(n=9)$ (Tabla 2).

El requerimiento de transfusiones fue de $26,4 \%$ ( $n=204$ ), el $66,6 \%$ fueron pacientes con neoplasias hematológicas $(n=136)$. En 762 pacientes se realizó el análisis de sobrevida global, en el grupo de pacientes sin anemia, la sobrevida global a los 12,36 y 60 meses fue estimada en $82,6 \%$, $68,4 \%$ y $62,0 \%$, respectivamente; y en el grupo con anemia en $67,2 \%, 54,0 \%$ y $47,0 \%$, respectivamente, con diferencia entre las curvas de sobrevida global de los pacientes sin anemia y con anemia $(p<0,001)$ (Figura 2$)$. En el grupo de pacientes con tumores sólidos, la sobrevida global en los casos sin anemia fue estimada a los 12,36 y 60 meses en $82,6 \%, 67,4 \%$ y $61,2 \%$, respectivamente; y en los casos con anemia en $66,6 \%, 55,0 \%$ y $49,0 \%$, respectivamente, con una diferencia entre las curvas de sobrevida global $(p=0,002)$ (Figura 3). Asimismo, en pacientes con tumores hematológicos, la sobrevida global en los casos sin anemia fue estimada a los 12,36 y 60 meses en $82,6 \%, 73,6 \%$ y $66,3 \%$, respectivamente; y en los casos con anemia en $68,5 \%, 52,3 \%$ y $43,4 \%$, respectivamente, con diferencia entre las curvas de sobrevida global $(p=0,007)$ (Figura 4).

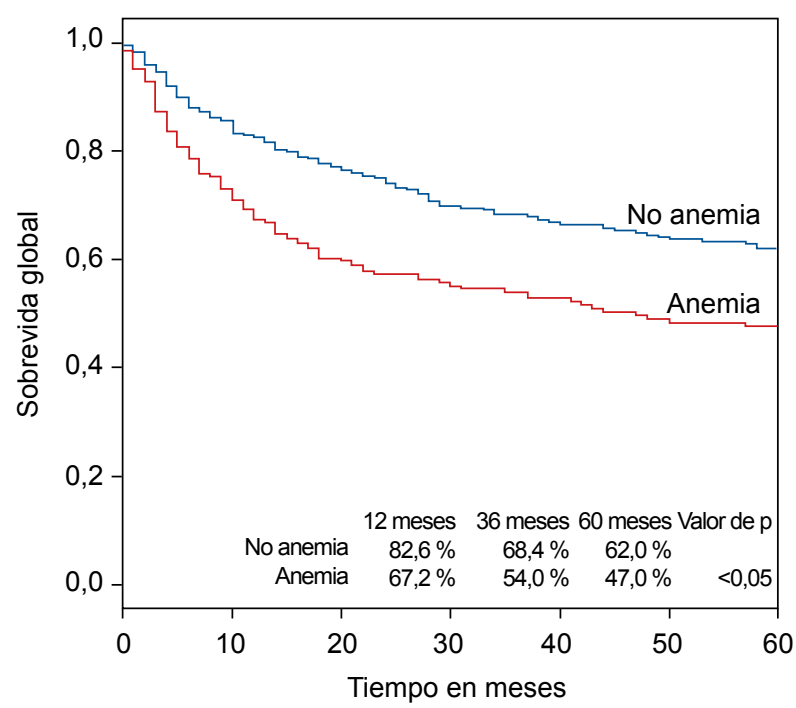

Figura 2. Curvas de sobrevida global según presencia de anemia en pacientes con cáncer del Instituto Nacional de Enfermedades Neoplásicas 
Tabla 1. Características clínicas y demográficas de pacientes con cáncer atendidos en el Instituto Nacional de Enfermedades Neoplásicas (INEN) entre enero y abril del 2010

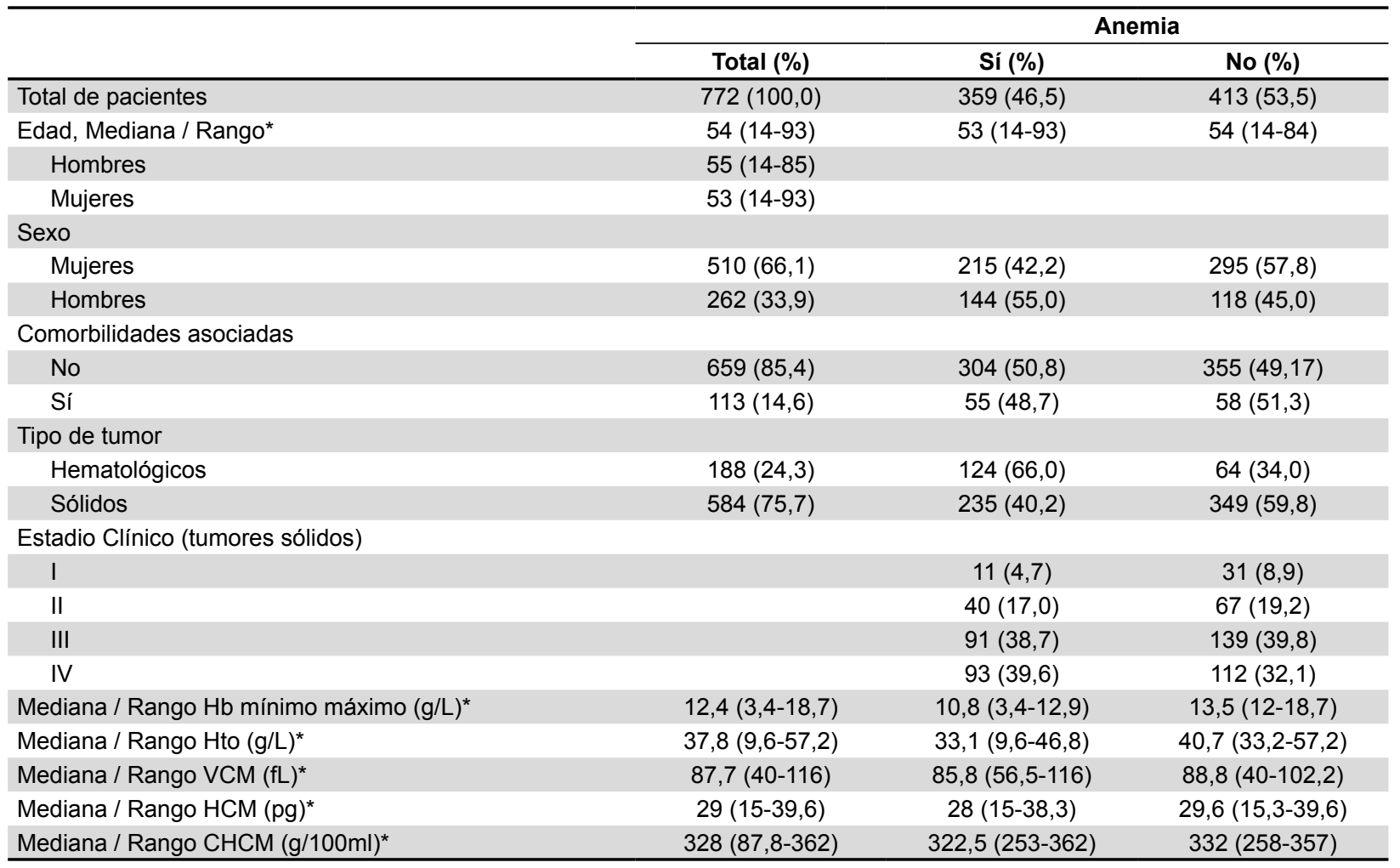

VCM: Volumen corpuscular medio, HCM: Hemoglobina corpuscular medio, CHCM: Concentración de hemoglobina corpuscular media.

* (Mínimo - máximo)

En el análisis multivariado, el sexo (HR: 1,8; IC 95\%: 1,4-2,3), la edad (HR: 1,6; IC 95\%: 1,3-2,1) y la anemia (HR:0,6; IC 95\%: 0,5-0,8) se muestran como importantes para explicar el tiempo hasta la muerte, en presencia de otras características. En los tumores sólidos, en el análisis multivariado el sexo pierde la significancia que tenía en el análisis crudo; sin embargo, se encontró que el estadio clínico III (HR: 18,4; IC 95\%: 2,5-133,1) y IV (HR: 76,3; IC
95\%: 10,6-551,5) influyen sobre el tiempo hasta la muerte (Tabla 3).

\section{DISCUSIÓN}

La frecuencia de anemia al ingreso entre pacientes diagnosticados con cáncer en el Instituto Nacional de Enfermedades Neoplásicas fue del 46,5\%. Los hallazgos

Tabla 2. Distribución de los niveles de anemia según origen del cáncer en pacientes del Instituto Nacional de Enfermedades Neoplásicas

\begin{tabular}{lcccccc}
\hline & \multicolumn{5}{c}{ Nivel de anemia (\%) } \\
\cline { 2 - 7 } Origen & Total (\%) & $\begin{array}{c}\text { Severa } \\
\mathbf{1 < 8} \mathbf{~ g / L )}\end{array}$ & $\begin{array}{c}\text { Moderada } \\
(\mathbf{8}-<\mathbf{1 1} \mathbf{~ g / L )}\end{array}$ & $\begin{array}{c}\text { Leve } \\
(\mathbf{1 1}-<\text { LNI g/L) }\end{array}$ & $\begin{array}{c}\text { Anemia total } \\
(<\mathbf{L N I} \text { g/L) }\end{array}$ & $\begin{array}{c}\text { Sin anemia } \\
(\mathbf{\geq} \text { al LNI g/L) }\end{array}$ \\
\hline Mama & $190(24,6)$ & $1(0,5)$ & $14(7,4)$ & $35(18,4)$ & $50(26,3)$ & $140(73,7)$ \\
Hematológico & $188(24,4)$ & $32(17,0)$ & $56(29,8)$ & $36(19,2)$ & $124(66,0)$ & $64(34,0)$ \\
Gastrointestinal & $137(17,7)$ & $10(7,3)$ & $38(27,7)$ & $37(27,0)$ & $85(62,0)$ & $52(38,0)$ \\
Ginecológico & $71(9,2)$ & $7(9,9)$ & $20(28,2)$ & $10(14,1)$ & $37(52,1)$ & $34(47,9)$ \\
Tumores de partes & $69(8,9)$ & $1(1,4)$ & $8(11,6)$ & $14(20,3)$ & $23(33,3)$ & $46(66,7)$ \\
blandas y sarcomas & $58(7,5)$ & - & $5(8,7)$ & $14(24,1)$ & $19(32,8)$ & $39(67,2)$ \\
Pulmón & $39(5,1)$ & - & $4(10,3)$ & $8(20,5)$ & $12(30,8)$ & $27(69,2)$ \\
Cabeza y cuello & $20(2,6)$ & $1(5,0)$ & $3(15,0)$ & $5(25,0)$ & $9(45,0)$ & $11(55,0)$ \\
Urológico & & & & & &
\end{tabular}

*LNI: limite normal inferior 


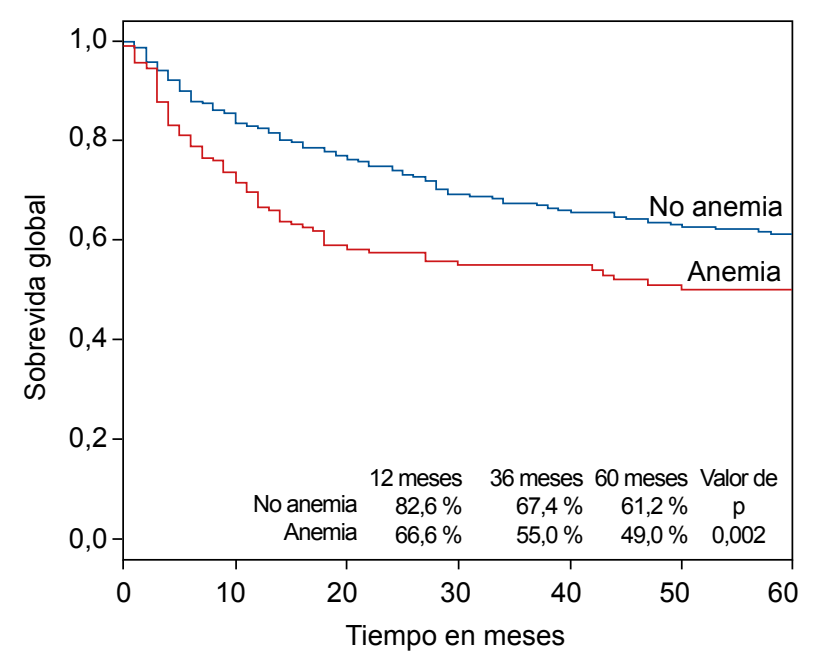

Figura 3. Curvas de sobrevida según presencia de anemia en pacientes con tumores sólidos del Instituto Nacional de Enfermedades Neoplásicas

se corroboran con la revisión realizada por Knight et al, y considera valores entre un 30 al $90 \%$ de acuerdo al tipo y estadio clínico, así mismo con la European Cancer Anaemia Survey (ECAS) que reveló una frecuencia de anemia en un $39 \%$ de la población estudiada ${ }^{(2,22)}$. Los puntos de corte para definir anemia son múltiples, y en algunos estudios inclusive fue el mismo según el sexo ${ }^{(2)}$. La mayor población fueron mujeres probablemente en relación a que de los cinco tipos de cáncer más frecuentes, tanto el cáncer de mama y cáncer de cérvix ocurren en mujeres, siendo un $29,8 \%$ de la población de estudio.

La anemia microcítica fue la más frecuente en el presente estudio, probablemente se debe a que el cáncer es

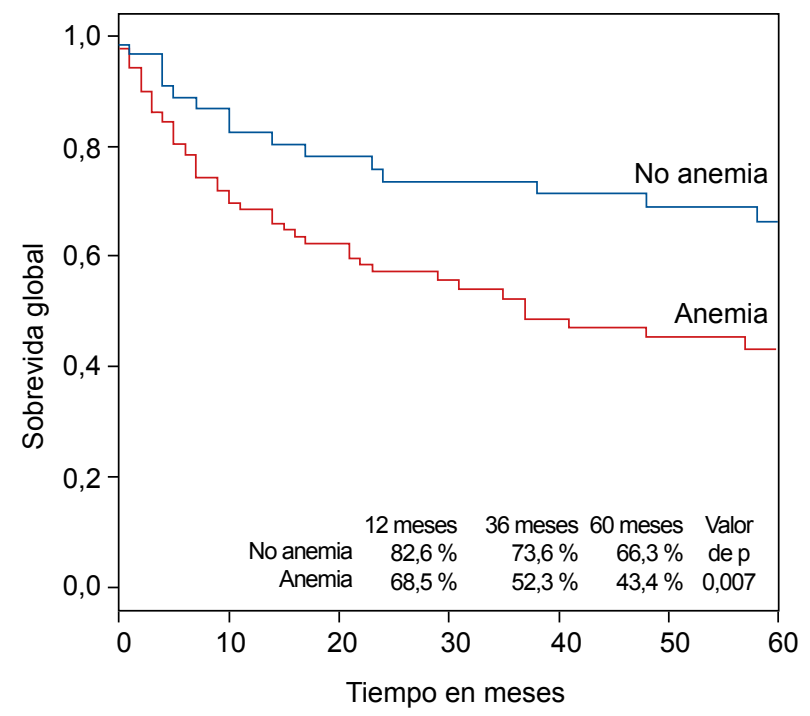

Figura 4. Curvas de sobrevida según presencia de anemia en pacientes con tumores hematológicos del Instituto Nacional de Enfermedades Neoplásicas una enfermedad crónica, y esto permita su ocurrencia mediante lo mecanismos fisiopatológicos previamente mencionados ${ }^{222)}$. La mayor proporción de pacientes tuvieron estadios avanzados de cáncer, sin embargo, la anemia leve fue la más frecuente; dicho hallazgo podría explicarse por los puntos de corte de hemoglobina empleados para determinar anemia, y por la ausencia de información sobre la procedencia del paciente, datos que permiten ajustar dichos puntos de corte.

Las neoplasias hematológicas que fueron más anemizantes (leucemia mieloide crónica, leucemias agudas y mieloma múltiple), tuvieron una menor cantidad de pacientes; por tanto, su frecuencia se justifica probablemente por su naturaleza y fisiopatología.

Así mismo las, neoplasias sólidas con mayor frecuencia de anemia fueron de origen gastrointestinal, seguidos de las de origen ginecológico y luego urológico, consideramos que en el último grupo se tuvo pocos casos. En el diferencial por neoplasias individuales, encontramos al cáncer de mama con una frecuencia de $26,3 \%$, ligeramente menor a lo reportado por el ECAS que obtuvo un $30 \%$ de frecuencia. El cáncer de pulmón tuvo una frecuencia del $25,5 \%$ mucho menor en relación a la ECAS quienes hallaron hasta un $38 \%$, probablemente en relación con el menor tamaño poblacional de nuestro estudio. En cuanto a tumores gastrointestinales se obtuvo una mayor frecuencia, tanto en el cáncer gástrico y colo-rectal, la ECAS obtuvo un $39 \%$ de frecuencia para tumores gastrointestinales en general. El cáncer de cérvix tuvo una frecuencia de $57,5 \%$, equiparable con el $55 \%$ y $67 \%$ hallados previamente ${ }^{(2,3)}$.

La incidencia de cáncer se incrementa en las últimas décadas de la vida, además la edad es considerada dentro de los índice de puntuación para pronóstico de sobrevida libre de progresión y sobrevida global; por ejemplo, el índice pronóstico internacional (IPI) para Linfomas No Hodgkin considera la edad de 60 años como punto de corte. Dichos aspecto explicarían la sobrevida en nuestro estudio.

El estadio clínico presentó asociación con una menor sobrevida, sin embargo, no se pudo determinar si la anemia fue un factor de riesgo independientemente de la severidad del cancer, o si la anemia es un modificador de la relación severidad - sobrevida.

Por último, la presencia de anemia refleja lo reportado en estudios previos, encontramos un incremento del $40 \%$ de la probabilidad de muerte, comparado al $65 \%$ en revisiones más grandes, esto podría fácilmente suponer que la anemia es un predictor de sobrevida, además sabemos que existen diferentes mecanismos complejos para la producción en anemia, sea por un efecto directo en la producción de 
Tabla 3. Análisis de factores pronósticos para sobrevida global en pacientes con cáncer en el Instituto Nacional de Enfermedades Neoplásicas

\begin{tabular}{|c|c|c|c|c|}
\hline & $\begin{array}{l}\text { HR crudo } \\
\text { (IC 95\%) }\end{array}$ & $\begin{array}{l}\text { Valor } \\
\text { de } p\end{array}$ & $\begin{array}{l}\text { HR ajustado } \\
\text { (IC } 95 \%)\end{array}$ & Valor de $p$ \\
\hline \multicolumn{5}{|c|}{ Todos los pacientes ( $n=772)$} \\
\hline \multicolumn{5}{|c|}{ Sexo } \\
\hline Mujeres & 1 & & 1 & \\
\hline Hombres & $1,9(1,5-2,4)$ & $<0,001$ & $1,8(1,4-2,3)$ & $<0,001$ \\
\hline \multicolumn{5}{|l|}{ Edad, años } \\
\hline$<60$ & 1 & & 1 & \\
\hline$\geq 60$ & $1,7(1,3-2,2)$ & $<0,001$ & $1,6(1,3-2,1)$ & $<0,001$ \\
\hline \multicolumn{5}{|l|}{ Comorbilidades } \\
\hline No & 1 & & 1 & \\
\hline Sí & $1,3(1,0-1,8)$ & 0,092 & $1,2(0,8-1,6)$ & 0,387 \\
\hline \multicolumn{5}{|l|}{ Tipo de tumor } \\
\hline Hematológico & 1 & & 1 & \\
\hline Sólido & $0,9(0,7-1,2)$ & 0,346 & $1,1(0,9-1,5)$ & 0,398 \\
\hline \multicolumn{5}{|l|}{ Anemia } \\
\hline Sí & 1 & & 1 & \\
\hline No & $0,6(0,5-0,8)$ & $<0,001$ & $0,6(0,5-0,8)$ & $<0,001$ \\
\hline \multicolumn{5}{|c|}{ Tipo Sólido ( $n=584)$} \\
\hline \multicolumn{5}{|c|}{ Sexo } \\
\hline Mujeres & 1 & & 1 & \\
\hline Hombres & $2,0(1,5-2,7)$ & $<0,001$ & $1,1(0,8-1,4)$ & 0,685 \\
\hline \multicolumn{5}{|l|}{ Edad, años } \\
\hline$<60$ & 1 & & 1 & \\
\hline$\geq 60$ & $1,6(1,2-2,2)$ & 0,001 & $1,5(1,1-2,0)$ & 0,010 \\
\hline \multicolumn{5}{|l|}{ Comorbilidades } \\
\hline No & 1 & & 1 & \\
\hline Sí & $1,3(0,9-1,8)$ & 0,25 & $1,0(0,7-1,5)$ & 0,998 \\
\hline \multicolumn{5}{|l|}{ Estadio Clínico } \\
\hline I & 1 & & 1 & \\
\hline II & $5,3(0,7-41,1)$ & 0,107 & $5,2(0,7-40,4)$ & 0,112 \\
\hline III & $19,3(2,7-138,9)$ & 0,003 & $18,4(2,5-133,1)$ & 0,004 \\
\hline IV & $80,1(11,2-575,8)$ & $<0,001$ & $76,3(10,6-551,5)$ & $<0,001$ \\
\hline \multicolumn{5}{|l|}{ Anemia } \\
\hline Sí & 1 & & 1 & \\
\hline No & $0,6(0,5-0,9)$ & 0,003 & $0,7(0,5-0,9)$ & 0,006 \\
\hline \multicolumn{5}{|c|}{ Tipo Hematológico ( $n=188)$} \\
\hline \multicolumn{5}{|c|}{ Sexo } \\
\hline Mujeres & 1 & & 1 & \\
\hline Hombres & $1,7(1,0-2,8)$ & 0,032 & $1,7(1,1-2,9)$ & 0,025 \\
\hline \multicolumn{5}{|l|}{ Edad, años } \\
\hline$<60$ & 1 & & 1 & \\
\hline$\geq 60$ & $1,9(1,2-3,0)$ & 0,009 & $1,9(1,1-3,0)$ & 0,012 \\
\hline \multicolumn{5}{|l|}{ Comorbilidades } \\
\hline No & 1 & & 1 & \\
\hline $\mathrm{Si}$ & $1,6(0,9-2,9)$ & 0,135 & $1,6(0,9-3,0)$ & 0,138 \\
\hline Anemia & & & & \\
\hline Sí & 1 & & 1 & \\
\hline No & $0,5(0,3-0,8)$ & 0,008 & $0,5(0,3-0,8)$ & 0,007 \\
\hline Anemia $(n=360)$ & & & & \\
\hline Sexo & & & & \\
\hline Mujeres & 1 & & 1 & \\
\hline Hombres & $2,1(1,5-3,0)$ & $<0,001$ & $2,0(1,4-2,8)$ & $<0,001$ \\
\hline Edad, años & & & & \\
\hline$<60$ & 1 & & 1 & \\
\hline$\geq 60$ & $1,8(1,3-2,5)$ & 0,001 & $1,6(1,1-2,2)$ & 0,011 \\
\hline Comorbilidades & & & & \\
\hline No & 1 & & 1 & \\
\hline $\mathrm{Si}$ & $1,5(1,0-2,4)$ & 0,063 & $1,3(0,8-2,0)$ & 0,310 \\
\hline Tipo de tumor & & & & \\
\hline Hematológico & 1 & & 1 & \\
\hline Sólido & $0,9(0,6-1,3)$ & 0,563 & $1,0(0,7-1,5)$ & 0,881 \\
\hline
\end{tabular}


hematíes o de manera indirecta por la carga tumoral y efectos biológicos, difíciles de determinar bajo un estudio observacional ${ }^{(3,10,23,24)}$.

El estudio se limita a pacientes de uno de los principales centros de manejo de cáncer dentro del Ministerio de Salud y en un determinado sitio geográfico (Lima), es difícil saber si esto refleja lo que pasa en el resto del país o demás sistemas de salud por lo que es necesario contar con más estudios que diluciden esta duda. Además, no fue diseñado para medir otras variables como niveles de hierro y otras deficiencias nutricionales, es importante mencionar que se debe realizar el diagnóstico de anemia como está establecido en las últimas guías clínicas. Se recomienda hacer esta evaluación cuando el paciente ingresa a un centro de atención del cáncer, debe incluir una adecuada anamnesis, recuento de reticulocitos, dosaje de hierro, ácido fólico, vitamina $\mathrm{B} 12$, saturación de transferrina y frotis de sangre periférica; y en caso sea necesario un estudio de médula ósea, así como la evaluación de la función renal ${ }^{(25)}$.

De acuerdo al resultado se debe clasificar los tipos de anemia relacionados a cáncer, esto permitirá un enfoque amplio y posteriormente tomar una conducta terapéutica, ya sea en base a transfusiones, uso de agentes estimulantes de la eritropoyetina, reposición de hierro, etc ${ }^{(5)}$. Otro punto importante sugiere que antes e inclusive durante el tratamiento es una necesidad para los médicos tratantes mantener unos niveles de hemoglobina adecuados; sin embargo, no se ha establecido en qué punto de corte se hace necesario realizar esta intervención, tampoco se puede definir un punto exacto donde la intervención tendrá efecto en el curso de la enfermedad, otros estudios han evaluado los niveles de hemoglobina a ser alcanzado por intervenciones terapéuticas para mejorar la sobrevida ${ }^{(12,25-27)}$.

En conclusión, la anemia relacionada al cáncer es una patología bastante frecuente en nuestra población, los diferentes mecanismos fisiopatológicos, factores epidemiológicos y clínicos muestran el complejo escenario en que se desarrolla. La anemia es un factor pronóstico independiente de sobrevida global. Hemos visto el rol de la anemia al inicio del tratamiento; sin embargo, aún queda por explicar varios aspectos respecto a la intervención con la seguridad de los agentes estimulantes de eritropoyetina, el rol del uso del hierro endovenoso, así como su frecuencia y efectos a largo plazo. Hacen necesarios futuros estudios prospectivos para confirmar estos hallazgos, así como las intervenciones necesarias que puedan mejorar la anemia en pacientes con cáncer.

Fuentes de financiamiento: Autofinanciado.

Conflictos de intereses: Ninguno de los autores tiene conflicto de intereses.

Contribuciones de autoría: VP, CA, ZM y LM conceptualizaron y diseñaron el artículo. VP, AL y GV recolectaron los datos. VP, CA, RM y ZM realizaron la interpretación de los datos. Todos los autores participaron en la redacción del artículo. ZM, TO y LM realizaron la revisión crítica del artículo. Todos los autores aprobaron la versión final del artículo.

\section{REFERENCIAS BIBLIOGRÁFICAS}

1. Organización Mundial de la Salud (Internet). Ginebra; c2017 (citado el 28 de noviembre del 2017). Concentraciones de hemoglobina para diagnosticar la anemia y evaluar su gravedad. Disponible en: http://www.who.int/vmnis/indicators/ haemoglobin_es.pdf.

2. Knight K, Wade S, Balducci L. Prevalence and outcomes of anemia in cancer: a systematic review of the literature. Am J Med. 2004; 116(7A):11S-26S.

3. Birgegård G, Aapro $M$ et al. CancerRelated Anemia: Pathogenesis, Prevalence and Treatment. Oncology 2005;68(suppl 1):3-11.

4. Ruiz R, Serrano M, Ruiz EF, Mantilla R, Valdivieso N, Olivera M, et al. Características clínico-patológicas y sobrevida en mujeres jóvenes con cáncer cervical: análisis retrospectivo del Instituto Nacional de Enfermedades Neoplásicas. Rev Peru Med Exp Salud Publica. 2017;34(2):218-27. doi: $10.17843 /$ rpmesp.2017.342.2653.
5. Gilreath A, Stenehjem D, Rodgers GM. Diagnosis and treatment of cancer-related anemia. Am J Hematol. 2014;89(2):20312. doi: $10.1002 /$ ajh.23628.

6. Mercadante S, Gebbia V, Marrazzo A, Filosto S. Anaemia in cancer: pathophysiology and treatment. Cancer Treat Rev. 2000;26(4):303-11.

7. Rizzo JD, Brouwers M, Hurley P, Seidenfeld J, Somerfield MR, Temin S. American Society of Clinical Practice Guideline Update on the use of Epoetin and Darboetin in Adult Patients with Cancer. J Oncol Pract. 2010;6(6):317-20. doi: 10.1200/JOP.2010.000132.

8. Flores-Balcázar C, Rosales-Pérez S, Galván-Salazar G, López-Navarro O. Anemia inducida por quimioterapia en pacientes oncológicos: papel de los agentes eritropoyéticos. Archivos de Medicina.2015;11(3):1-11

9. Organización Mundial de la Salud. Ginebra; c2017 (citado el 30 de noviembre del 2017). Worldwide prevalence of anemia 1993-2005. WHO global database on anemia 1993-2005. Disponible en:http://apps.who.int/iris/bitstre am/10665/43894/9789241596657_eng. $\mathrm{pdf}$ ?sequence $=1$.

10. Caro JJ, Salas M, Ward A, Goss G. Ane$\mathrm{mia}$ as an independent prognostic factor for survival in patients with cancer: a systemic, quantitative review. Cancer. 2001;91(12):2214-21.

11. Zhang Y, Chen Y, Chen D, Jiang Y, Huang W, Ouyang $\mathrm{H}$, et al. Impact of preoperative anemia on relapse and survival in breast cancer patients. BMC Cancer. 2014;14:844. doi:10.1186/14712407-14-844.

12. Littlewood T. The impact of hemoglobin levels on treatment outcomes in patients with cancer. Semin Oncol. 2001; 28(suppl 8):49 -53 .

13. Lind $M$, Vernon C, Cruickshank D, Wilkinson P, Littlewood T, Stuart N, et al. The level of haemoglobin in anaemic cancer patients correlates positively with 
quality of life. Br J Cancer. 2002 Apr 22;86(8):1243-9.

14. Holzner B, Kemmler G, Greil R, Kopp $\mathrm{M}$, Zeimet A, Raderer $\mathrm{M}$, et al. The impact of hemoglobin levels on fatigue and quality of life in cancer patients. Ann Oncol. 2002;13(6):965-73.

15. Groopman J, Itri L. Chemotherapy-Induced Anemia in Adults: Incidence and Treatment. J Natl Cancer Inst. 1999;91(19):1616-34.

16. Sabbatini P. The Relationship between Anemia and Quality of Life in Cancer Patients. Oncologist. 2000;5 Suppl 2:19-23.

17. Ferrucci L, Balducci L. Anemia of aging: the role of chronic inflammation and cancer. Semin Hematol. 2008;45(4):242-9. doi: $10.1053 /$ j.seminhematol.2008.06.001. doi:10.1053/j.seminhematol.2008.06.001.

18. Zhu W, Xu B. Association of Pretreatment Anemia with Pathological Response and Survival of Breast Cancer Patients Treated with Neoadjuvant Chemotherapy: A Population-Based Study. PLoS One. 2015;10(8):e0136268. doi: 10.1371/journal.pone.0136268.

19. Hasenclever D, Diehl V. A prognostic score for advanced Hodgkin's disease.
International Prognostic Factors Project on Advanced Hodgkin's Disease. N Engl J Med. 1998;339(21):1506-14.

20. Greer J, Arber D, Glader B. Wintrobe's Clinical Hematology. 13th edition. Philadelphia, PA: Lippincott Williams \& Wilkins, a Wolters Kluwer bussiness.

21. Edge S, Byrd D, Compton C, Fritz A, Greene F, Trotti A. AJCC cancer staging manual. 7th ed. New York, NY: Springer; 2010.

22. McLean E, Cogswell M, Egli I, Wojdyla $\mathrm{D}$, de Benoist B. Worldwide prevalence of anaemia, WHO Vitamin and Mineral Nutrition Information System, 1993-2005. Public Health Nutr. 2009;12(4):444-54. doi: $10.1017 /$ S1368980008002401.

23. Yang Y,Li T,Nielsen ME. Aging and cáncer mortality: dynamics of change and sex differences. Exp Gerontol. 2012;47(9):695705. doi: 10.1016/j.exger.2012.06.009.

24. Zhou Z, Sehn LH, Rademaker AW, Gordon LI, Lacasce AS, Crosby-Thompson A, et al. An enhanced International Prognostic Index (NCCN-IPI) for patients with diffuse large B-cell lymphoma treated in the rituximab era. Blood. 2014;123(6):837-42. doi: 10.1182/blood-2013-09-524108.
25. Bokemeyer C, Aapro MS, Courdi A, Foubert J, Link H, Osterborg A, et al. EORTC Guidelines for the use of Erythropoietic Proteins in Anaemic Patients with Cancer: 2006 update. Eur J Cancer. 2007;43(2):258-70.

26. Coiffier B, Guastalla J, Pujade-Lauraine E, Bastit P, Anemia Study Group. Predicting cancer-associated anaemia in patients receiving non-platinum chemotherapy: results of a retrospective survey. Eur $\mathrm{J}$ Cancer. 2001;37(13):1617-23.

27. NCCN Clinical Practice Guidelines Cancer-and chemotherapy induced anemia. Ver 2.2017 (Internet). USA (Citado el $17 \mathrm{de}$ diciembre del2017). Disponible en: https:// www.nccn.org/professionals/physician_gls/ pdf/anemia.pdf.

Correspondencia: Victor Roman Paitan Amaro Dirección: Jr. Lloque Yupanqui 937. Dpto 403.

Jesús Maria. Lima, Perú.

Teléfono: +51922639373

Correo electrónico: victorpaitana@gmail.com 